\title{
Civilisations
}

Revue internationale d'anthropologie et de sciences

humaines

$44 \mid 1997$

Les peuples des forêts tropicales

\section{Culture, nature, nationalisme et internationalisme}

L'exemple des Yanomami et des Peuples du Bassin Amazonien

\section{Pierrette Birraux-Ziegler}

\section{(2) OpenEdition}

\section{Journals}

Édition électronique

URL : http://journals.openedition.org/civilisations/1621

DOI : $10.4000 /$ civilisations. 1621

ISSN : 2032-0442

Éditeur

Institut de sociologie de l'Université Libre de Bruxelles

\section{Édition imprimée}

Date de publication : 1 janvier 1997

Pagination : 126-137

ISBN : 2-87263-122-4

ISSN : 0009-8140

\section{Référence électronique}

Pierrette Birraux-Ziegler, "Culture, nature, nationalisme et internationalisme », Civilisations [En ligne], 44 | 1997, mis en ligne le 29 juin 2009, consulté le 19 avril 2019. URL : http://journals.openedition.org/ civilisations/1621 ; DOI : 10.4000/civilisations. 1621 
CULTURE, NATURE, NATIONALISME

ET INTEANATIONALISME:

l'exemple des Yanomami el

des Peuples du Bassin Amazonien

Pierrette BIPRAUX-Zl|EGLER 


\section{LES FAUX OLLEMMES}

L'humanisme occidental a consacré l'opposition entre culture et nature, une opposition qui n'a guère de sens pour les peuples autochtones du monde. Plus récemment, les polémiques suscitées par la destruction des forêts tropicales se sont souvent basées sur de semblables dichotomies, autant du côté des conservationnistes que de celui des destructeurs de la forêt. Pour ces derniers, la reconnaissance des droits ancestraux des Indiens d'Amazonie et la préservation de la forêt «s'opposent» tour à tour au «développement» du pays ou à sa "sécurité nationale». Les environnementalistes, de leur côté, parviennent difficilement à accepter que des ethnies non occidentales, sans pouvoir être qualifiées d'écologistes (ce serait absurde!), ont développé une longue convivialité avec les écosystèmes qu'ils prétendent protéger.

En voulant ignorer que les rapports humains à la nature varient dans le temps et dans l'espace, les institutions internationales conservationnistes, relayées par leurs consoeurs nationales, ont imposé aux populations locales une conception de la protection de l'environnement née des prédations provoquées par l'industrialisation et relevant d'une cosmologie européenne et anglosaxonne. Il en résulte de grandes injustices à l'égard des peuples autochtones expropriés de leurs territoires ancestraux par la création de réserves et de parcs naturels. Or, ces territoires ont été et sont encore choisis par les environnementalistes précisément parce que leurs habitants traditionnels les ont bien conservés! Cette contradiction de fond se traduit sur le terrain par de nombreux et cinglants échecs quant à la mise en pratique de ces mesures, échecs recensés par Col- chester [1994]. Au point qu'aujourd'hui, les conservationnistes sont amenés à reconnaître que la préservation de la nature est impossible sans l'active participation des populations locales, surtout autochtones, telles qu'en témoignent les résolutions de la dernière assemblée générale de l'Union internationale pour la conservation de la nature (UICN : résolution 19.20 et recommandations 19.21 et 19.22); reconnaissance qui n'a pas encore passé dans la pratique car elle suppose une remise en question fondamentale d'ordre philosophique et politique ainsi que l'élaboration de nouvelles méthodologies et de nouveaux outils. Je pense notamment à l'instauration de dialogues interculturels basés sur le respect et l'égalité des parties soit les représentants gouvernementaux, les environnementalistes et les communautés et/ou organisations autochtones. De tels dialogues sont aisés à préconiser mais beaucoup plus difficiles à réaliser!

Une autre opposition simpliste sert souvent d'argument pour éviter de reconnaître aux peuples autochtones la propriété communautaire de leurs territoires et également pour contrer ceux qui se préoccupent de protection de l'environnement. Il s'agit de l'argument nationaliste (qui fonctionne dans tous les cercles politiques, de quelque obédience qu'ils soient), selon lequel la reconnaissance des territoires autochtones et la protection de l'environnement viseraient à empêcher les États du Sud de se "développer». Comme nous venons de le voir, si l'idéologie conservationniste (qui ne constitue qu'un type d'interventions à but écologique parmi d'autres) peut se convertir en actions néo-colonialistes, il faut aussi rappeler que de nombreux États du Sud siègent 
dans les instances décisionnelles de l'UiCN et du WWF International, pour ne citer que ces deux principales organisations diffusant le conservationnisme. Ces États se sont montrés, en règle générale, plus soucieux de suivre ces organismes que d'écouter les premiers habitants de leur propre pays. D'où la tendance des Autochtones d'aller eux-mêmes chercher, au niveau international, une reconnaissance qu'ils ne parviennent pas à obtenir dans leur propre pays.

\section{L'HITTORE RÉCENTE DES YANOMAMI ECHAPPE AUX DURULSMES SIMPLISTES}

L'exemple des Yanomami - ce peuple forestier vivant aux confins du Brésil et du Venezuela et pratiquant l'agriculture sur brûlis, la chasse, la pêche et la cueillette - montre combien ces oppositions simplistes ne tiennent guère face aux réalités historiques et actuelles. En effet, les premiers non-Indiens à s'intéresser à cette ethnie restée isolée jusque dans les années 50 ont été des missionnaires, des scientifiques et des aventuriers européens et nord-américains, ayant généralement joui d'appuis officiels dans les deux pays. De plus, la campagne visant à éviter la disparition physique et culturelle des Yanomami, menée depuis les années 70 par une $0 \mathrm{NG}$ brésilienne, verrait son efficacité réduite à peu de choses sans les nombreux relais qu'elle a su mobiliser dans les États industrialisés. Son but fondamental n'est pourtant que de convaincre le gouvernement de ce pays de respecter sa propre Constitution, celleci reconnaissant aux Indiens leurs territoires ancestraux. Ce faisant, cette ONG a mis en œuvre les mêmes moyens que ceux utilisés par la société civile pendant la dictature militaire, lorsque sa seule planche de salut était d'alerter l'opinion publique internationale des exactions commises à l'encontre des leaders paysans, syndicalistes et étudiants.

La présence missionnaire en territoire yanomami brésilien et vénézuélien constitue un autre exemple montrant combien l'opposition entre national et étranger est factice. Au Venezuela, la loi des missions datant de 1915 ne donne-t-elle pas aux religieux - pendant longtemps des Européens et des Nord-anéricains - les pleins pouvoirs sur les zones qu'ils occupent? Et ce, en dépit des exigences de l'article 2 de la Constitution du pays qui prescrit qu'une authentique loi autochtone doit être élaborée?

Les adeptes des Nouvelles Tribus et autres sectes évangéliques fondamentalistes d'origine nord-américaine - les premiers à établir des contacts permanents avec les Yanomami - n'ontils pas été appelés et conduits par les Forces aériennes brésiliennes aux frontières même du pays, frontières encore mal définies et où n'existait aucune autre présence non yanomami? Cette dernière «alliance» s'est d'ailleurs montrée particulièrement durable : en 1987 - soit près de 30 ans après les premières pénétrations missionaromilitaires - seuls les évangélistes sont autorisés à rester en territoire yanomami, lorsque conmence l'invasion massive de celui-ci par des dizaines de milliers de chercheurs d'or. En revanche, médecins, missionnaires catholiques et scientifiques sont expulsés par le gouvernement, qu'ils soient brésiliens ou étrangers. Parallèlement, au Venezuela, le fort mouvement national des années 80 contre les agissements destructeurs de ces mêmes évangélistes à l'égard des cultures indiennes n'a 
pas abouti. Le ministre de la Justice de l'époque en décida autrement, ce qui a amené de nombreux Vénézuéliens à croire qu'une intervention étrangère était à l'origine du maintien de la secte.

Actuellement, il y a 10 postes missionnaires au Brésil et 11 au Venezuela pour environ 360 communautés ou 24.000 personnes, dispersées sur un territoire d'env. $190.000 \mathrm{~km}^{2}$. Contrairement à ce que celà laisse supposer, leur impact spatial et donc écologique n'est pas négligeable. Disposant de pistes d'atterrissage, d'objets manufacturés et parfois d'équipement sanitaire, ils provoquent une sorte de «satellisation» des établissements indiens voire leur sédentarisation. Ils constituent ainsi une première étape vers l'agglomération des communautés en des aires limitées, contraire à la structure traditionnelle d'occupation territoriale yanomami, faite de dispersion et de mobilité. Au Brésil, l'impact spatial des religieux cadre donc bien avec l'objectif militaire de création de "pôles de développement» à l'intérieur du territoire yanomami, "pôles» qui doivent permettre le démembrement de la plus grande partie de celui-ci et son occupation, par la société nationale.

Dans ce pays, les principales agressions frontales auquelles les Yanomami ont à faire face sont connues. D'origine surtout nationale, leurs auteurs ont parfois joui de complicités étrangères, volontaires ou non. De 1973 à 76, la construction, au Sud du territoire yanomami, de la route périmétrale Nord, une parallèle septentrionale de la Transamazonienne, provoque quelques centaines voire un millier de morts parmi les Indiens. Promue par le gouvernement militaire, elle bénéficie de crédits internationaux jusqu'à ce que le financeur apprenne qu'elle sert plus à des objectifs stratégiques que de développement économique. En 1975-76, le haut plateau de Surucucu est envahi par environ 500 chercheurs de minerais précieux, suite à la publication des conclusions du projet Radambrasil. Ce vaste inventaire des ressources amazoniennes mené par le Brésil en coopération avec les États-Unis localise en effet dans cette région de l'or, de la cassitérite, des minerais radioactifs et «stratégiques». Les conflits qui en résultent chassent les missionnaires nordaméricains et obligent le gouvernement fédéral à retirer les envahisseurs. Un poste «d'attraction» de la Fondation nationale de l'Indien (FUNAI) y est établi en 1976, un quartier militaire en 1987 et un poste de la Police fédérale en 1990.

Les intérêts qui suscitent et profitent des invasions des chercheurs d'or sont essentiellement nationaux. Plus étonnant est le constat fait par une équipe de géologues et d'anthropologues brésiliens selon lequel il en est de même des intérêts des compagnies minières. En effet, seuls $9 \%$ des concessions octroyées ou demandées en territoire yanomami le sont par des firmes multinationales soit Brascan et Anglo-American Simonsen. Elles ne couvrent que 7\% de la superficie de toutes les concessions demandées et/ou octroyées. En revanche, $81 \%$ sont le fait d'entreprises privées nationales et $10 \%$ d'entreprises étatiques. L'ensemble de ces demandes et 
octrois concerne $34 \%$ du territoire yanomami [CEDI, 1988].

Si de 1968 à 1979, anthropologues et missionnaires avaient présenté une douzaine de propositions de délimitation du territoire yanomami au gouvernement brésilien, en vue de sa protection, c'est essentiellement dès la $2^{\mathrm{e}}$ moitié des années 70 que la scène internationale est régulièrement sollicitée en faveur de ce peuple.

En 1978 est fondée la Commission pour la création du parc yanomami (CCPY), une ONG brésilienne qui se charge d'abord de diffuser de l'information fiable concernant la situation des Yanomami, de plaider en faveur du parc (selon la terminologie juridique de l'époque), puis, d'apporter des soins de santé aux Yanomami. En 1980, l'«Anthropology Ressource Center» (ARC) de Washington présente le cas au 4e Tribunal Russel qui conclut à une situation proche du génocide. La même année, la situation dramatique

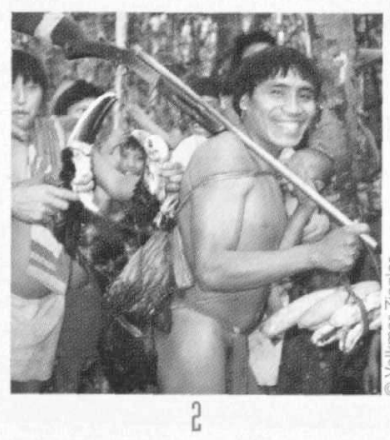
des Yanomami est soumise par cinq ONG et la Société brésilienne d'anthropologie à la Commission inter-américaine des Droits de l'Homme de l'Organisation des États américains (OEA). En 1981, une publication de ARC alerte la communauté internationale tandis que la première intervention aux Nations-Unies, à la Sous-commission sur la prévention de la discrimination et la protection des minorités, est réalisée par la Société anti-esclavage de Londres. Par la suite, chaque année, de semblables interventions seront faites par diverses organisations non gouvernementales (ONG) y compris amérindiennes. La plupart se basent sur des sources brésiliennes ou sont rédigées par des spécialistes brésiliens - indiens ou non indiens -

Face à ces interventions, l'État brésilien se sent contraint d'agir c'est-à-dire d'appliquer sa propre législation. En 1982, il interdit le territoire yanomami, premier pas administratif en direction de sa délimitation et de sa protection. Trois ans plus tard, il le délimite sur carte (mais pas sur le terrain). Simultanément, les militaires - qui ont toujours été opposés à la reconnaissance légale des territoires indiens - élaborent en secret le Projet calha norte. Rendu public par une indiscrétion en 1986, ce programme d'occupation militaire des frontières septentrionales du pays comprend des mesures d'assistance aux communautés locales tout en étant essentiellement motivé par des considérations stratégiques. Ses résultats sont connus : invasion dès 1987 du territoire yanomami par des dizaines de milliers de chercheurs de minerais précieux - or et cassitérite -; 15 à $20 \%$ de la population yanomami du Brésil meurt en deux ans et demi (d'août 1987 à janvier 1990) à la suite de maladies ou de violences; transgression des frontières suscitant le militarisation de la partie vénézuélienne du territoire yanomami; pollution des rivières au mercure et destruction de pans entiers de forêt provoquant la malnutrition des Indiens.

Les campagnes nationales et internationales redoublent. L'assemblée générale de l'Organisation internationale du travail (OIT) accuse le Brésil de violer la Convention 107 sur les populations 
indigènes et tribales signée par ce pays. En réponse, le gouvernement invite la présidente du Groupe de travail sur les populations autochtones (G'I'PA) des Nations-Ininies à se rendre chez les Yanomami. En 1988, le prix Global du Programme des Nations-Unies pour l'environnement est attribué à Davi Yanomami, porte-parole d'un peuple dont il est l'un des rares ressortissants parlant le portugais. Celui-ci est alors reçu par Perez de Cuellar, Secrétaire général de l'ONI!.

le discrédit jeté sur le Brésil, à propos notamment de la déforestation abusive de l'Amazonie et de la violence à l'encontre des mouvements populaires y compris indiens, fait le reste. À la veille de la Conférence de Rio sur l'environnement et le développement, le président Collor homologue le territoire yanomami. La plus grande partie des chercheurs d'or est retirée... mais pas tous. leurs dirigeants attendent que s'éteignent les projecteurs qui illuminent l'ancienne capitale du pays pour reprendre, discrètement, leurs invasions, encouragés par les prises de position des autorités locales et la passivité des militaires. Ies maladies continuent à sévir chez les Yanomami sans que les services de santé nationaux sachent se montrer à la hauteur. Au moment où j'écris ces lignes (novembre 1994), la situation est dramatique tant au Brésil qu'au Venezuela, les envahisseurs transgressant la frontière sans que les forces armées n'interviennent de manière convaincante.

\section{Un dialogue à inventer : celui entre les Élals. les conservationnistes et les Hutochtones}

Au Venezuela, l'histoire récente des Yanomami présente des différences évidentes par rap- port à celle du Brésil. Si les premiers contacts sont semblables à ceux connus par leurs frères brésiliens - expéditions scientifiques étrangères, commissions frontalières, incursions périphériques de créoles et établissement de missionnaires évangélistes et catholiques soutenus par l'État - les Yanomami du Venezuela n'ont pas connu d'agression frontale et massive.

La pénétration non indienne a été plus graduelle et jusqu'à présent, l'ex-territoire fédéral d'Amazonas, devenu récemment État, est protégé de facto car ne figurant pas (encore?) parmi les régions que le gouvernement désire exploiter ou coloniser en priorité. Il n'en est toutefois pas de même de l'État de Bolivar où vivent les Indiens Sanimá, un sous-groupe yanomami, qui subit une acculturation accélérée suscitée par l'exploitation minière pratiquée chez leurs voisins Panare et Pemón.

Au début des années 70 , le gouvernement vénézuélien - inquiet des motivations géopolitiques préludant au programme amazonien des militaires brésiliens - lance son propre projet de "Conquista del Sur», une conquête vite stoppée par le président Carlos Andres Perez. Vite stoppée car le pays ne répond ni en termes d'investissements, ni de mouvements migratoires. Contrairement au Brésil, le slogan «sécurité et développement" ne marche pas dans ce pays vivant dans une prospérité et une démocratie relatives. Prospérité et démocratie qui permettent aux Vénézuéliens de ne pas systématiquement recourir à la communauté internationale lorsque les droits des Indiens sont menacés. Ainsi, les concessions minières octroyées chez les Yanomami, aux sources de l'Orénoque, sont retirées en 1982, 
essentiellement grâce aux pressions internes, de l'Église catholique notamment.

Une autre différence notoire entre le Venezuela et le Brésil concernant les peuples autochtones est que le premier pays n'a développé aucune loi indigéniste contrairement à ses obligations constitutionnelles. En outre, le seul instrument international concernant les Autocthones qu'il ait signé, la Convention 107 de l'OI'T, a certes été ratifiée par le Parlement mais cette ratification n'a jamais été communiquée à Genève. Elle est donc inopérante. De fait, il règne au Venezuela une sorte de jacobinisme qui a beaucoup de peine à reconnaître la spécificité indienne tant au niveau légal que pratique.

En revanche, la législation environnementaliste y est pléthorique. In tiers du territoire vénézuélien fait l'objet d'une administration spéciale de la part de l'État pour des raisons environnementales. Beaucoup de ces parcs nationaux, réserves écologiques, réserves forestières et autres monuments naturels sont situés en terre indienne puisqu'elle est la mieux préservée. Ici, c'est par le biais de la protection de l'environnement que l'influence des organisations internationales se fait principalement sentir. Et cette influence est possible parce qu'au Venezuela, et particulièrement en Amazonas, le couple «sécurité et développement» s'étant révélé inefficace, l'État lui a substitué celui de «sécurité et environnement» pour y renforcer son autorité.

Dans un tel contexte, la figure juridique et environnementaliste de "réserve de la biosphère" a paru être la seule possible pour protéger le territoire yanomami, ses habitants et les sources de l'Orénoque, suite aux violations de frontières per- pétrées par les orpailleurs brésiliens dès 1988. Ie fait même que cette mesure ait été prise peu après leurs premières incursions montre bien que c'est le territoire national que l'on veut d'abord protéger. En effet, deux autres propositions très semblables, élaborées par des anthropologues, existaient depuis le début des années 80 et n'avaient jamais fait l'objet d'une décision.

La création d'une réserve de la biosphère pose deux problèmes aux Autochtones. D'abord, une mainmise étatique sur des territoires dont les autorités ne se sont guère préoccupées auparavant, lorsqu'il s'agissait essentiellement de dispenser des soins de santé; d'autre part, la quasiimpossibilité, lorsqu'une telle mesure est adoptée, d'une reconnaissance du droit de propriété foncière collective de ses habitants. Par définition en effet, la réserve de la biosphère ne leur reconnaît qu'un droit d'usage, jouissance et usufruit (uso, goze y disfrute)

La composition de la Commission permanente de gestion semble confimer cette mainmise étatique : administrée par un Secrétariat autonome de l'État d'Amazonas, elle comprend six ministères, trois autres organismes étatiques, deux institutions académiques, le Vicariat apostalique de Puerto Ayacucho, un représentant de la société civile nommé par le Ministère de l'environnement et des représentants yanomani et yekuana. La réserve n'étant toutefois pas encore aménagée, le sort de ses habitants dépend du dialogue que chacun des acteurs sera capable d'instaurer avec eux.

À ce décret s'en ajoute un deuxième, qui érige en parc national la moitié orientale de la surface de la réserve de la biosphère. En raison de ses qualités paysagères, une fonction culturelle, 
éducative et récréative lui est attribuée, dont on peut se demander à qui elle est destinée.

D'autres peuples indiens du Venezuela ont déjà des expériences bien négatives des parcs nationaux taillés à même leur territoire. Les Pemón se sont vu interdire de pratiquer leurs activités traditionnelles de subsistance telles que la chasse, la pêche, la cueillette et l'agriculture sur brûlis dans le parc Canaima tandis qu'ils doivent y supporter la présence de «touristes environnementalistes». S’ils veulent survivre, il ne leur reste que le braconnage, la profession de «guide touristique» ou celle - éminemment prédatrice écologiquement et culturellement - de chercheur d'or dans les placers situés juste au nord du parc! ̀̀ une autre extrémité du pays, les Yucpa et les Bari expérimentent actuellement comment l'Institut des parcs bat vite en retraite lorsqu'une entreprise étatique comme Corpozulia veut exploiter les ressources minières du

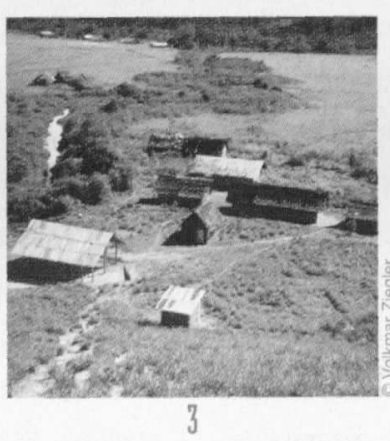

transformait en un nouvel instrument de spoliation territoriale.

En conséquence, pour que la réserve de la biosphère constitue une mesure véritablement efficace quant à la survie des Yanomami et aussi à leur «autogestion» et «autodéveloppement» termes employés dans le décret de création de la réserve - il importe que leurs organisations soient écoutées au sein de la Commission permanente de gestion, de même que l'Organisation régionale des peuples indiens d'Amazonas (ORPIA) et le Conseil national indien du Venezuela (CONIVE).

Le CONIVE a, en effet, rédigé - suite à une consultation des communautés indiennes au niveau national - un projet de «Loi organique de protection des ethnies, communautés et citoyens autochtones» qui comprend une figure juridique de reconnaissance de leurs droits territoriaux qui synthétise les impératifs indigénistes et environnementalistes. Il s'agit de la parc national de la Sierra Perijá.

Ces deux exemples - et tous ceux analysés par Colchester dans l'article déjà cité - confirment une réflexion faite par une équipe de recherche de l'Université de Montréal [Décarie et al., 1977] dont je faisais partie, au milieu des années 70 . En analysant la politique des parcs et des réserves pratiquée aux États-Unis dès la deuxième moitié du XIXe siècle, nous en avions conclu que celle-ci nuisait davantage aux populations locales, surtout amérindiennes, qu'aux activités prédatrices qu'elle prétendait combattre. Au mieux, elle servait à les différer dans le temps. Au pire, elle se
«Zone de protection autochtone» où il est clairement spécifié : «Dans tous les cas, le régime des zones de protection autochtone aura la préférence sur n'importe quel autre régime spécial de pro-

Les Indiens ont fait le premier pas et la balle est dans le camp des autorités. Si celles-ci savent garantir aux Indiens leurs droits légitimes - surtout celui à la terre - et instaurer un dialogue exempt de préjugés avec eux, alors, une chance existe de concilier préservation de la nature et ethno-développement, sans que la communauté internationale ait à se mobiliser. tection environnementale». 


\section{LE ROOLE DE LR COMMUHAUTE ENTERHATIONHLE}

Nous venons de voir que cette dernière s'est manifestée chez les Yanomami dès les premiers contacts et ce, de manière diversifiée et contradictoire, qu'elle a toujours collaboré avec ses interlocuteurs nationaux - qu'il s'agisse d'ONG ou de gouvernements - et que souvent, ces derniers se sont servis des crédits qu'elle octroie et des règles qu'elle a élaborées pour mettre en pratique leurs propres ohjectifs nationaux y compris ceux relatifs à la sécurité nationale.

L'utilisation d'arguments nationalistes pour éviter de garantir les droits des Indiens d'Amazonie n'a donc guère de cohérence par rapport à l'histoire récente de la politique des fítats à leur égard, surtout dans des pays comme le Brésil ou le Venezuela, où ceux-ci sont fortement minoritaires et entendent le concept d'autodétermination comme étant le droit de perpétuer leur culture et leur mode de vie à l'intérieur des sociétés nationales. Malgré ces évidences, ces deux pays adoptent, dans les forums internationaux, une attitude très négative à l'égard des peuples autochtones, comme on peut le constater lors des sessions annuelles du GTPA des Nations-Unies. Tandis que le Chili et l'Argentine, en voie de démocratisation comme le Brésil, adoptent une attitude plus souple, le géant lusitanien reste aussi rigide qu'à l'époque de la dictature et, je serais tentée de dire, le Venezuela qu'au temps de la démocratie!

Au niveau international, les Autochtones disposent de très peu d instruments garantissant leur spécificité. En fait, les seules Conventions contraignantes pour les États signataires sont celles du BIT soit les Conventions 107 et 169 , la seconde étant la révision de la première qui date de 1957 et est jugée paternaliste par les Autochtones.

Si le Projet de déclaration des droits des peuples autochtones, élaboré conjointement par les représentants autochtones et ceux des États dans le cadre du GTPA de l'ONU, comporte aujourd'hui les termes-clés d'autodétermination et de peuple, il risque d'être fortement altéré au fur et à mesure qu'il gravira les échelons que représentent la Commission des Droits de l'Homme, le Conseil économique et social (ECOSOC) et l'Assemblée générale, où le pouvoir de décision revient actuellement uniquement aux États. En effet, le Projet de déclaration reconnaît de manière explicite que les Autochtones constituent des peuples et non des populations. Dans la mesure où la Convention internationale sur les droits civils et politiques stipule que : «Tous les peuples ont le droit à l'autodétermination. En vertu de ce droit, ils déterminent librement leur statut politique et poursuivent librement leur développement économique, social et culturel ", quelques États comprenant des Autochtones à l'intérieur de leurs frontières (et dont le Brésil est un ténor) refusent de leur reconnaître un statut de peuple. On en est ainsi arrivé à la situation absurde - mais qui montre bien le rapport de force entre Autochtones et États au niveau des organisations internationales - de la Convention 169 datant de 1989 qui emploie bien le mot "peuple» mais ajoute immédiatement au 3e paragraphe de l'article 1 : "L'emploi du terme "peuple» daus la présente convention ne peut en aucune manière être interprété comme ayant des implications de quelque nature que ce soit quant aux droits qui peuvent s'attacher à ce terme en 
vertu du droit international». En d'autres termes, les peuples autochtones n'ont toujours pas les mêmes droits que les autres peuples et surtout pas celui à l'autodétermination, même lorsque celuici est conçu comme valable uniquement à l'intérieur des frontières nationales [Eide, 1994].

Mais il y a d'autres canaux que les organisations autochtones peuvent utiliser au niveau inter-gouvernemental et ce sont eux que la Coordination des organisations indiennes du bassin amazonien (COICA) a privilégiés jusqu'à présent. Cette organisation faitière des organisations indiennes des 9 pays du bassin amazonien peut être considérée comme leur bras diplomatique.

Elle ne s'est pas contentée de participer aux discussions politiques dont je viens de faire état. Pragmatique, elle a cherché à participer à d'autres instances. La première est la Commission spéciale pour les affaires autochtones du Traité de coopération amazonienne dont elle a

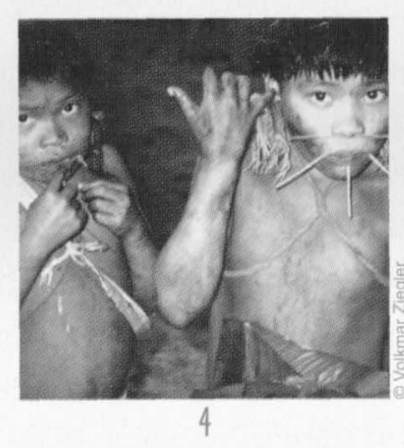

culté à passer à la pratique. Ainsi, lorsque la Coordination des organisations indiennes de l'Amazonie brésilienne (COIAB) a demandé son appui pour la démarcation des terres indiennes du Bassin, la Banque mondiale a posé comme préalable l'extinction des prétentions des Autochtones sur deux territoires pour lesquels ils luttaient depuis des années.

La COICA s'est aussi alliée aux principales organisations environnementalistes afin que celles-ci reconnaissent la participation des Autochtones à la sauvegarde des écosystèmes. Il en est résulté la déclaration d'Iquitos de 1990 dont l'article premier déclare que la protection de la forêt amazonienne passe par la garantie légale de leurs terres aux Indiens.

Finalement, la COICA a promu une alliance avec plus de 350 villes européennes qui s'engagent : à réduire leurs émissions de $\mathrm{CO}^{2}$ dans l'atmosphère afin de diminuer l'effet de serre; à exercer des pressions sur leurs gouvernements lorsque des banques ou des entreprises de leur pays planifient des projets contraires aux intérêts des Autochtones et de la forêt amazonienne; et à consacrer une partie de leur budget de coopération au développement à des projets émanant des communautés indiennes amazoniennes, dont il est reconnu qu'elles jouent un rôle protecteur de la forêt.

On vient de le voir, plusieurs portes se sont déjà ouvertes au niveau international pour que les organisations autochtones puissent s'exprimer et promouvoir des solutions originales quant au mondiale a répondu favorablement à leur demande même si elle a encore bien de la diffiavec ces organismes de coopération multilatérale, afin qu'ils écoutent les communautés et organisations indiennes avant de financer les projets que les gouvernements leur destinent ou destinent à la mise en valeur de leurs ressources. La Banque demande mêne si elle a encore bien de la diffi- 
développement et la protection de l'environnement dans leur région, notions qui n'ont rien de contradictoire pour elles, pas plus que le fait de recourir aux instances internationales pour se faire entendre aux niveaux national et local. Pour elles, le travail doit être réalisé simultanément aux trois niveaux pour atteindre une quelconque efficacité à moyen et long terme. Il importe aussi que les populations nationales prennent conscience que les sociétés autochtones ont beaucoup à apporter au développement de leur pays, à la protection des milieux naturels et à la formation de véritables identités nationales, enracinées dans le continent sud-américain. 


\section{LÉGENOEES DES PHOTOS}

1: Abattis yanomami.

¿ : Retour de chasse dans la Sierra Parima, Venezuela. La chair cuite du toucan et du nocco sera accompagnée de bananes plantains cuites.

3: Destruction de l'habitat traditionnel yaromani, conséquence de l'action missionnaire des Nouvelles tribus - Sierra Parima, Venezuela.

4: Récolte de miel sauvage. 